\title{
Oxidative Stress and Antioxidant Status in Immune Thrombocytopenia
}

\author{
Xuewei Li ${ }^{1}$, Zhaoyue Wang ${ }^{1 *}$, Xiaofei Qi ${ }^{1}$ and Dongjie Zhang ${ }^{2}$ \\ ${ }^{1}$ Jiangsu Institute of Hematology, Collaborative Innovation Center of Hematology, Soochow University, Suzhou, China \\ ${ }^{2}$ Qingdao Municipal Hospital, Qingdao, China
}

"Corresponding author: Zhaoyue Wang, The First Affiliated Hospital of Soochow University, Jiangsu Institute of Hematology, A Key Laboratory of Thrombosis and Hemostasis of Ministry of Health, Collaborative Innovation Center of Hematology, Soochow University, Suzhou, China, Tel: +86-512-67780872; E-mail: zwang11@sina.com

Received date: July 21, 2017; Accepted date: September 28, 2017; Published date: October 07, 2017

Copyright: ( $2017 \mathrm{Li} \mathrm{X}$, et al. This is an open-access article distributed under the terms of the Creative Commons Attribution License, which permits unrestricted use, distribution, and reproduction in any medium, provided the original author and source are credited.

\begin{abstract}
Immune thrombocytopenia (ITP) is an autoimmune disorder characterized by an isolated thrombocytopenia that may manifest as skin and mucous membrane bleeding. However, the pathogeny of this disease is much elusive. Increasing evidences demonstrate oxidative stress plays an essiential role in the pathogenesis of autoimmune diseases including ITP, which may provide a novel therapeutic approach.
\end{abstract}

Keywords: Lupus erythematosus; Reactive oxygen; Pathogenesis; Autoimmune inflammatory disease

\section{Introduction}

Immune thrombocytopenia (ITP) is an autoimmune disorder characterized by isolated thrombocytopenia due to pathogenic antiplatelet autoantibodies, $\mathrm{T}$ cell-mediated platelet destruction, and impaired megakaryocyte function [1]. This disorder is classified as primary, also referred to as idiopathic thrombocytopenic purpura, or as secondary to an underlying disorder such as chronic infections, including Helicobacter pylori, or other autoimmune diseases including systemic lupus erythematosus (SLE) and antiphospholipid syndrome [2]. Many aspects of immune dysregulation in ITP have widely been investigated, but its precise mechanisms are still unclear, and the exact triggering events remain elusive. Recently, several evidences have suggested that oxidative stress plays an important role in the pathogenesis of autoimmune diseases, and it is also involved in the development of ITP. These findings might provide a new hypothesis for the initiation of abnormal autoimmunity and a possible novel therapeutic approach.

\section{Oxidative stress and antioxidant status}

Reactive oxygen species (ROS) could be generated from normal cellular metabolism such as oxidative phosphorylation in mitochondria and long-chain fatty acids oxidation in peroxisomes, and be served as second messengers [3]. The ROS mainly include oxygen molecule $\left(\mathrm{O}_{2}\right)$, superoxide anion radical $\left(\bullet^{-}{ }^{-}\right)$, hydrogen peroxide $\left(\mathrm{H}_{2} \mathrm{O}_{2}\right)$, hydroxyl radical $(\bullet \mathrm{OH})$, and singlet oxygen $\left(1 \mathrm{O}_{2}\right)$ [4]. On the other hand, in maintaining the equilibrium, there also exist antioxidants and antioxidant enzymes such as superoxide dismutase (SOD), glutathione peroxidase $\left(\mathrm{GP}_{\mathrm{x}}\right)$ and catalase (CAT), which are responsible for regulating the redox state. Mitochondria and lysosomes are considered to be the two important subcellular organelles that mediate oxidative stress. In general, the forms of life maintain a reducing environment within their cells, preserved by several relevant enzymes. In general, at low to moderate concentrations, ROS act as important molecules involved in many physiological processes such as cell activation, viability, proliferation, and organ function, and they also play important roles in inflammatory response and anti-infection, as they can be used by the immune system to attack and kill pathogens [5]. Oxidative stress occurs as a result of increased activity of free radical-producing enzymes, decreased activity of free radicalremoving enzymes, and insufficient levels of antioxidants [6]. It may cause irreversible changes such biomolecules as lipids, proteins and carbohydrates. On the other hand, it can also induce a variety of different types of DNA damage or replication stress [7]. It has been demonstrated that oxidative stress in a hypoxia re-oxygenation reaction in endothelial cells may induce profound damage, reflecting chromosomal aberrations and micronuclei [8]. Thus, ROS is the production of normal cells and plays an important role in the metabolism of our body. However, if it is generated excessively or unable to be eliminated, it will be harmful to our health.

\section{Oxidative stress and autoimmunity}

Increased oxidative stress has been proved to be related to the development of a variety of diseases such as atherosclerosis [9], neurodegenerative diseases [10] and cancer [3]. Moreover, it also plays a vital role in the pathogenesis of autoimmune diseases. Oxidant stress could induce autoimmune dysregulation, which includes the generation of neo-epitopes and auto-antigens, leading to B- and T-cell dysregulation and the generation of autoantibodies (Figure 1). It has been reported that, in patients with autoimmune cholestatic liver diseases (AC) and autoimmune hepatitis (AIH), the levels of all lipid and protein oxidative injury products are increased, while the whole blood glutathione levels are significantly decreased. Furthermore, protein carbonyl and isoprostane levels are increased and glutathione levels decreased gradually with progression from mild fibrosis to severe fibrosis and cirrhosis in both $\mathrm{AC}$ and $\mathrm{AIH}$ patients. These findings support a major contribution of oxidant/antioxidant imbalance in the progression of liver injury in autoimmune liver diseases [5]. SLE is an autoimmune inflammatory disease whose pathogenesis also refers to oxidative stress. Several studies have shown increased production of ROS or diminished levels of intracellular reduced glutathione in various blood components in SLE patients $[11,12]$. Several investigators suggested that restoration of the redox balance using antioxidant agents or diminishing effect of oxidative stress by intake of antioxidant nutrients, might attenuate the 
complication of SLE induced by oxidative stress [11]. Similarly, the deficiencies of SOD and peroxiredoxin inducing oxidative stress were considered to be an essential factor that might participate in the pathogenesis of another autoimmune disease autoimmune hemolytic anemia. The SOD1-deficient mice model showed a positive correlation between autoantibodies and ROS in RBCs. RBCs are under oxidative stress constitutively, and cellular components face the risk of oxidative damage [13]. Endothelial inflammation, mitochondrial oxidative stress would be activated in the condition of high glucose levels. Therefore, type 2 diabetes (T2D) would be served as a prime example of an interplay between metabolism and immunity, making it prototypic for an in depth look into immunometabolism [14-16]. Growing evidence implies that immune cells possess an essential effect on the development of insulin resistance and then complications of T2D [17-19]. Numerous investigators have observed elevated levels of oxidative stress markers in patients with T2D [20,21]. Therefore, the imbalance between oxidant and antioxidant systems is also involved in the pathogenesis of T2D and its complications [22-24]. Recent data demonstrates that oxidative stress influences cell-cycle regulators in $\beta$ cell proliferation and neogenesis [25]. Furthermore, several lines of evidence suggest that oxidative stress is implicated in the pathogenesis of diabetic neuropathy (DSPN) [26]. Many researchers have observed that oxidative stress is increased in peripheral and dorsal root ganglion nerves and vascular endothelial cells exposed to hyperglycemia $[26,27]$. And for T2D patients with DSPN, the total antioxidant status was decreased compared with controls $[28,29]$.

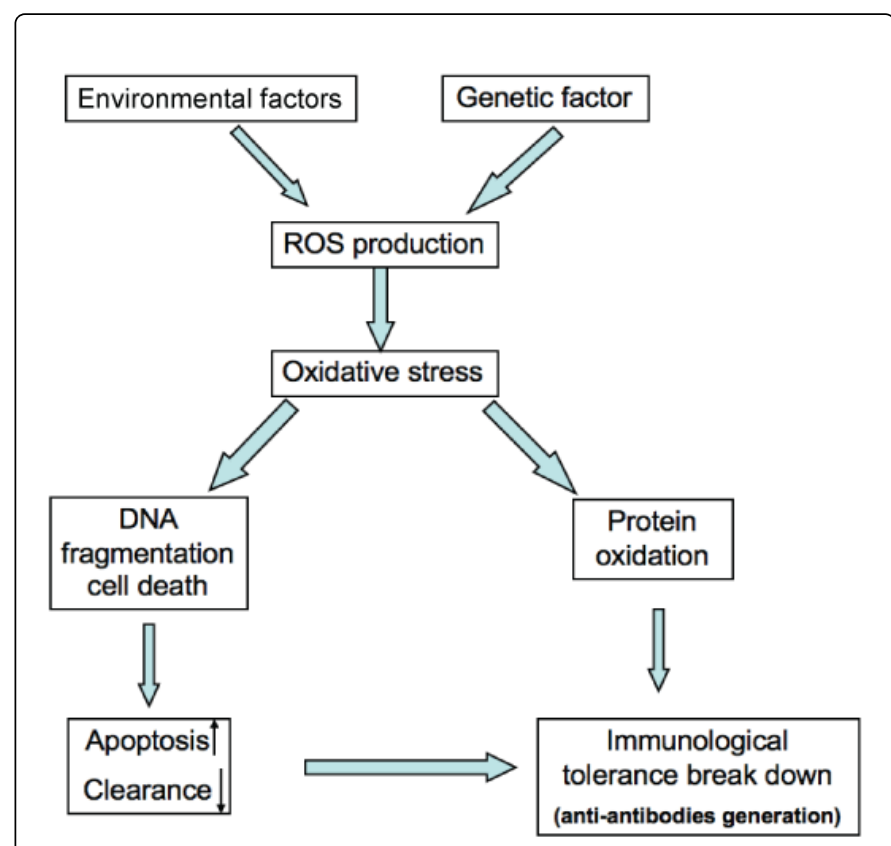

Figure 1: Oxidative stress involved in the pathogenesis of autoimmune disorders.

\section{Oxidative stress in ITP}

ITP is an autoimmune disease due to autoantibodies against one or several platelet surface antigens, but the mechanism is complex. In the past decade, many evidences have confirmed that oxidative stress was involved in the pathogenesis of ITP. Polat et al. [30] studied lipid peroxidation, glutathione, and ascorbic acid levels in adult ITP patients, and they found that lipid peroxidation level was higher, and glutathione and ascorbic acid levels were lower in the patient group than in the control group, which implies that oxidative damage is involved in the mechanism of ITP. Other researchers also confirmed lipid peroxidation, reduced glutathione (GSH) and oxidative stressrelated pathways in whole blood and/or plasma samples from ITP patients $[31,32]$. Recently, a more profound method of microarray analysis has been used as a tool to explore the gene expression profiles of patients with acute and chronic ITP. The expression of the gene vanin-1 was increased in patients with chronic ITP during the acute phase and patients with treatment-resistant ITP [33]. As regard to different phases of ITP, the markers present differently, the antioxidant capacity of ITP patients in the active phase was drastically reduced, while higher GPx activity was observed in both active phase and remission in comparison to healthy controls [34]. Interestingly, investigators found that female patients with ITP demonstrated significantly higher oxidative stress than male patients, suggesting sex differences in the pathomechanisms of ITP [34]. With regard to antioxidant status and oxidative stress in chronic ITP in adult patients, Jin et al. [35] found that the number of platelets had a negative correlation with NO, oxidized glutathione(GSSG), malondialdehyde (MDA), and total oxidant status (TOS), respectively, while platelet number showed a positive correlation with SOD, CAT, GPx and GSH. It has been found that markers of antioxidant status were significantly lower in patients with ITP compared to healthy controls. In addition, MDA was significantly higher in patients with newly diagnosed ITP compared to both healthy controls and patients with chronic ITP [31]. All these observations almost approved that oxidative stress may be partially responsible for the maintenance in thrombocytopenia.

\section{Antioxidant therapy for autoimmune disorders}

Oxidative stress, characterized by a persistent imbalance between the production of highly ROS and reactive nitrogen species and antioxidant defence leads to an altered cellular redox status and subsequent tissue damage. A growing body of researches have demonstrated that the etiology and pathogenesis of autoimmune diseases may be closely related to the unbalance between pro-oxidation and antioxidant. Therefore, many experts hold the idea of using antioxidants to eliminate excessive oxidizing agent. Numerous therapies for oxidative stress related autoimmune disorders have been tried, including corticosteroids, biologics, antibiotics, immunosuppresive drugs, 5-aminosalicylates and vitamin D analogs [36]. As anti-oxidants serving to limit relevant damage, alpha lipoic acid (ALA) shows a clear metabolic influence, which could improve microcirculation, and has an anti-inflammatory effect, and it could contribute to the defense against oxidative stress by increasing the synthesis of anti-oxidants like glutathione, one of the most abundant intra-cellular anti-oxidants in the body. Furthermore, there is growing evidence that ALA is beneficial for improving insulin resistance for T2D patients, and for improving microcirculation [37]. However, some other experts considered these therapies are mostly directed towards providing temporary symptomatic relief. Thus many studies have been drifted towards approaches to mitigate this damage by supplementing antioxidant enzymes such as SOD, CAT and glutathione peroxidase $[37,38]$, which could inhibit depolymerization of hyaluron of synovial fluid, inhibiting degradation of neutrophils and proteoglycans in articular cartilage, preventing collagen fragmentation by scavenging reactive oxidants, reducing elastase activity and inhibiting lipid peroxidation [38]. Nevertheless, various reports suggest they have poor pharmacokinetic profile that limits their use. The most common 
therapeutic approaches for ITP are corticosteroids, immunosuppressive drugs or splenectomy. Unfortunately, certain adult patients could not remain in remission, and no other regimens are universally effective. In this condition, some physicians focused on the role of oxidant stress in the pathophysiology of ITP and study relevant drugs. There has been reported that the use of ascorbic acid in refractory ITP seems effective and might be an attractive therapeutic option for patients who were resistant to some or all of the usual therapy [39]. Elalfy et al. [40] reported adjuvant antioxidant therapy containing vitamin $\mathrm{A}, \mathrm{C}$ and $\mathrm{E}$ and selenium yeast could significantly decrease MDA in children and adolescents with ITP, which might be considered as a complementary therapy with detectable oxidative stress to improve bleeding score as well as platelet count. However, there existed some controversial results. For instance, Steven and Bierling concluded eventually that ascorbic acid is not very effective in patients with refractory ITP [41,42]. Meanwhile, for children and adolescents who were diagnosed with ITP, Akbayram et al. [43] did not find significant differences in the oxidative stress and antioxidant capacity following steroid treatment. While Cura et al. [44] demonstrated an increase in the mean total antioxidant status level, and a decrease in the mean total oxidant status and oxidative stress index levels following one week of high-dose methylprednisolone treatment in children ITP. In addition, it has been proved an inhibitory effect of dexamethasone and prednisolone on the generation of reactive oxygen species from platelets, and this effect of both drugs was dose-dependent.

\section{Conclusion}

Oxidative modification of proteins and defect in $\mathrm{T}$ cell signal processing and/or antigen presentation could elicit antibodies in a variety of autoimmune diseases including ITP, in which antioxidant therapy has been demonstrated to ameliorate the oxidative stress. Standard therapy of ITP comprises corticosteroids, immunosuppressive agent, intravenous immunoglobulin, and splenectomy. However, some patients fail to respond to standard treatment or relapse. Recently, antioxidant therapeutic approach has shown to raise platelet count, indicating the value of adjunctive therapy. Further large scale prospective study will confirm its effectiveness in treating these patients with ITP.

\section{Conflicts of Interest}

We declare that we have no conflicts of interest.

\section{Grant}

Fund by The Project of Invigorating Health Care through Science, Technology and Education, Jiangsu Provincial Key Medical Center and the Priority Academic Program Development of Jiangsu Higher Education Institutions (PAPD).

\section{References}

1. Zufferey A, Kapur R, Semple JW (2017) Pathogenesis and therapeutic mechanisms in immune thrombocytopenia (ITP). J Clin Med 6: 16.

2. Liebman HR, Stasi R (2007) Secondary immune thrombocytopenic purpura. Curr Opin Hematol 14: 557-573.

3. Useros MJ, Li WY, Morales CM, Foncillas GJ (2017) Oxidative stress: A new target for pancreatic cancer prognosis and treatment. J Clin Med 6: 29.
4. Lima ALF, Melo JT, Silva AE, Oliveira AH, Timoteo AR, et al. (2012) DNA damage by singlet oxygen and cellular protective mechanisms. Mutat Res 751: 15-28.

5. Kaffe ET, Rigopoulou EI, Koukoulis GK, Dalekos GN, Moulas AN (2015) Oxidative stress and antioxidant status in patients with autoimmune liver diseases. Redox Report 20: 33-41.

6. Kurien BT, Scofield RH (2008) Autoimmunity and oxidatively modified autoantigens. Autoimmun Rev 7: 567-573.

7. Cadet J, Ravanat JL, Porro TM, Menoni H, Angelov D (2012) Oxidatively generated complex DNA damage: tandem and clustered lesions. Cancer Lett 327: 5-15.

8. Bresgen N, Karlhuber G, Krizbai I, Bauer H, Bauer HC, et al. (2003) Oxidative stress in cultured cerebral endothelial cells induces chromosomal aberrations, micronuclei, and apoptosis. J Neurosci Res 72: 327-333.

9. Li H, Horke S, Förstermann U (2014) Vascular oxidative stress, nitric oxide and atherosclerosis. Atherosclerosis 237: 208-219.

10. Patel VP, Chu CT (2014) Nuclear transport, oxidative stress, and neurodegeneration. Int J Clin Exp Pathol 4: 215-229.

11. Perl A (2013) Oxidative stress in the pathology and treatment of systemic lupus erythematosus. Nat Rev Rheumatol 9: 674-686.

12. Shah D, Sah S, Wanchu A, Wu MX, Bhatnagar A (2013) Altered redox state and apoptosis in the pathogenesis of systemic lupus erythematosus. Immunobiology 218: 620-627.

13. Fujii J, Kurahashi T, Konno T, Homma T, Iuchi Y (2015) Oxidative stress as a potential causal factor for autoimmune hemolytic anemia and systemic lupus erythematosus. World J Nephrol 4: 213-222.

14. Assar ME, Angulo J, Manas RL (2016) Diabetes and ageing-induced vascular inflammation. J Physiol 594: 2125-2146.

15. Scuteri A, Cunha PG, Rosei EA, Badariere J, Bekaert S, et al. (2014) Arterial stiffness and influences of the metabolic syndrome: a crosscountries study. Atherosclerosis 233: 654-660.

16. Sell H, Habich C, Eckel J (2012) Adaptive immunity in obesity and insulin resistance. Nat Rev Endocrinol 8: 709-716.

17. Hoefer IE, Steffens S, Korpela AM, Back M, Badimon L, et al. (2015) Novel methodologies for biomarker discovery in atherosclerosis. Eur Heart J 36: 2635-2642.

18. Nishimura S, Manabe I, Nagasaki M, Eto K, Yamashita H, et al. (2009) CD8+ effector T cells contribute to macrophage recruitment and adipose tissue inflammation in obesity. Nat Med 15: 914-920.

19. Scheiermann C, Frenette PS, Hidalgo A (2015) Regulation of leucocyte homeostasis in the circulation. Cardiovasc Res 107: 340-351.

20. Tiwari BK, Pandey KB, Abidi AB, Rizvi SI (2013) Markers of oxidative stress during diabetes mellitus. J Biomark 3:1-8.

21. Robertson RP (2004) Chronic oxidative stress as a central mechanism for glucose toxicity in pancreatic islet beta cells in diabetes. J Biol Chem 279: 42351-42354.

22. Pan HZ, Zhang L, Guo MY, Sui H, Li H, et al. (2010) The oxidative stress status in diabetes mellitus and diabetes nephropathy. Acta Diabetol 47: 71-76.

23. Odum EP, Ejilemele AA, Wakwe VC (2012) Antioxidant status of type 2 diabetic patients in Port Harcourt, Nigeria. Niger J Clin Pract 15: 55-58.

24. Shi YC, Pan TM (2012) Red mold, diabetes, and oxidative stress: A review. Appl Microbiol Biotechnol 94: 47-55.

25. Wang JJ, Wang HJ (2017) Oxidative stress in pancreatic beta cell regeneration. Oxid Med Cell Longev 9.

26. Bandeira MS, Fonseca LJ, Guedes SG, Rabelo LA, Goulart MO, et al. (2013) Oxidative stress as an underlying contributor in the development of chronic complications in diabetes mellitus. Int J Mol 14: 3265-3284.

27. Busui PR, Sima A, Stevens M (2006) Diabetic neuropathy and oxidative stress. Diabetes Metab Res Rev 22: 257-273.

28. Kasznicki J, Kosmalski M, Sliwinska A, Mrowicka M, Stanczyk M, et al. (2012) Evaluation of oxidative stress markers in pathogenesis of diabetic neuropathy. Mol Biol 39:8669-8678. 
Citation: Li X, Wang Z, Xiaofei Q, Zhang D (2017) Oxidative Stress and Antioxidant Status in Immune Thrombocytopenia. J Blood Disord Transfus 8: 391. doi:10.4172/2155-9864.1000391

Page 4 of 4

29. Merzouk S, Hichami A, Madani S, Merzouk H, Berrouiguet AY, et al (2003) Antioxidant status and levels of diferent vitamins determined by high performance liquid chroma-tography in diabetic subjects with multiple complications. Gen Physiol Biophys 22: 15-27.

30. Polat G, Tamer L, Tanriverdi K, Gürkan E, Baslamisli F, et al. (2002) Levels of malondialdehyde, glutathione and ascorbic acid in idiopathic thrombocytopenic purpura. East Afr Med J 79: 446-449.

31. Akbayram S, Doğan M, Akgün C, Mukul Y, Peker E, et al. (2010) The association of oxidant status and antioxidant capacity in children with acute and chronic ITP. J Pediatr Hematol Oncol 32: 277-281.

32. Zhang B, Lo C, Shen L, Sood R, Jones C, et al. (2011) The role of vanin-1 and oxidative stress-related pathways in distinguishing acute and chronic pediatric ITP. Blood 117: 4569-4579.

33. Milz KJ, Bal G, Sterzer V, Milz KS, Arbach O, et al. (2012) Reduced antioxidant capacities in platelets from patients with autoimmune thrombocytopenia purpura (ITP). Platelets 23: 184-194.

34. Milz KJ, Salama A (2014) Oxidative stress is predominant in female but not in male patients with autoimmune thrombocytopenia. Oxid Med Cell Longev 2014 1-9.

35. Jin CQ, Dong HX, Cheng PP, Zhou JW, Zheng BY, et al. (2013) Antioxidant status and oxidative stress in patients with chronic ITP Scand J Immunol 77: 482-487.

36. Serafim G, Navarro SA, Zarpelon AC, Ribeiro PFA, Fattori V (2015) Bosentan a mixed endothelin receptor antagonist, inhibits superoxide anion-induced pain and inflammation in mice. Naunyn Schmiedebergs Arch Pharmacol 388: 1211-1221.
37. Poh ZX, Goh KP (2009) A current update on the use of alpha lipoic acid in the management of type 2 diabetes mellitus. Endocr Metab Immune Disord Drug Targets 9: 392-398.

38. Bao B, Mitre C, Wijesinghe P, Marchetti L, Girsch E, et al. (2017) Treating triple negative breast cancer cells with erlotinib plus a select antioxidant overcomes drug resistance by targeting cancer cell heterogeneity, Scientific Report 7: 44125

39. Brox AG, Jan HK, Fauser AA (1988) Treatment of idiopathic thrombocytopenic purpura with ascorbate. Br J Haematol 70: 341-344.

40. Masugi J, Iwai M, Kimura S, Ochi F, Suzuki K, et al. (1994) Combination of ascorbic acid and methylprednisolone pulse therapy in the treatment of idiopathic thrombocytopenic purpura. Intern Med 33: 165-166.

41. Jubelirer SJ (1993) Pilot study of ascorbic acid for the treatment of refractory immune thrombocytopenic purpura. Am J Hematol 43: 44-46.

42. Codeau B, Bierling P (1990) Treatment of chronic autoimmune thrombocytopenic purpura with asorbate. Br J Haematol 75: 289-290.

43. Elalfy MS, Elalfy MS, Elhenawy YI, Deifalla S, Hegazy M, et al. (2015) Oxidant/Antioxidant status in children and adolescents with immune thrombocytopenia (ITP) and the role of an adjuvant antioxidant therapy. Pediatr Blood Cancer 62: 830-837.

44. Cura M, Koç A, Aksoy N, Özdemir ZC (2016) Effect of short-term, highdose methylprednisolone on oxidative stress in children with acute immune thrombocytopenia. Blood Res 51: 261-267. 